Laura J. Bonnett, PhD Catrin Tudur Smith, PhD Sarah Donegan, $\mathrm{PhD}$ Anthony G. Marson, PhD

Correspondence to Prof. Marson: A.G.Marson@liverpool.ac.uk

Supplemental data at Neurology.org

\section{Treatment outcome after failure of a first antiepileptic drug}

OPEN

\section{ABSTRACT}

Objectives: We assessed the likelihood of 12-month seizure remission and treatment failure after failure of a first antiepileptic drug, and identified factors influencing these outcomes.

Methods: SANAD (Standard and New Antiepileptic Drug) was a randomized controlled trial comparing monotherapy with standard and new antiepileptic drugs. Patients were followed up to study completion, even if they were switched from their randomized treatment. After a first treatment failure, we assessed the probability of 12-month seizure remission and treatment failure. Prognostic modeling identified predictors of these outcomes.

Results: Forty-four percent of patients in the SANAD trial had a first treatment failure. Seventy-five percent of these subsequently achieved 12-month remission by 6 years of follow-up. Significant prognostic factors included sex, age at treatment failure, time on randomized treatment at treatment failure, neurologic insult, total number of tonic-clonic seizures at treatment failure, reason for treatment failure, seizure type, and CT/MRI scan result. After a first treatment failure, young patients without tonic-clonic seizures, with a normal CT/MRI scan and failing their treatment because of unacceptable adverse events, had the highest likelihood of 12-month remission. Approximately $50 \%$ of patients who failed a first treatment also failed their second. Significant prognostic factors included total number of tonic-clonic seizures at first treatment failure, reason for first treatment failure, and CT/MRI scan result. Patients with tonic-clonic seizures and failing because of inadequate seizure control had the highest risk of a second treatment failure.

Conclusions: A high proportion of patients will achieve 12-month remission after a first treatment failure. Clinical factors can stratify patients according to likely outcome. Neurology ${ }^{\circledR}$ 2014;83:552-560

\section{GLOSSARY}

AED $=$ antiepileptic drug; SANAD = Standard and New Antiepileptic Drug.

Sixty to seventy percent of patients with epilepsy will achieve a remission from seizures, the majority doing so on their first antiepileptic drug (AED). ${ }^{1-3}$ After a first treatment failure, the clinician and patient will discuss the likely overall prognosis and decide on the next intervention. Currently, there is only limited evidence regarding the likely outcome after a first treatment failure in epilepsy. Following the International League Against Epilepsy classification of refractory epilepsy, ${ }^{4}$ there have been studies attempting to identify risk factors for drug resistance ${ }^{5}$; however, they do not provide estimates that are easily applied to a specific individual, or stratified for groups of similar individuals.

Prognostic models are rare in epilepsy and include those derived from the National General Practice Survey of Epilepsy, ${ }^{6}$ the MRC Antiepileptic Drug Withdrawal Study, ${ }^{7,8}$ the Multicentre Study of Early Epilepsy and Single Seizures, ${ }^{9}$ and models for patients with focal and generalized epilepsy derived from the Standard and New Antiepileptic Drug (SANAD) trial. ${ }^{10,11}$

SANAD arm A recruited 1,721 patients (89\% focal epilepsy) who were randomized to treatment with carbamazepine, gabapentin, lamotrigine, topiramate, or oxcarbazepine. SANAD arm B

From the Departments of Biostatistics (L.J.B., C.T.S., S.D.) and Molecular and Clinical Pharmacology (A.G.M.), University of Liverpool, UK. Go to Neurology.org for full disclosures. Funding information and disclosures deemed relevant by the authors, if any, are provided at the end of the article. The Article Processing Charge was paid by the University of Liverpool, from NIHR programme grant RP-PG-0606-1062.

This is an open access article distributed under the terms of the Creative Commons Attribution-Noncommercial No Derivative 3.0 License, which permits downloading and sharing the work provided it is properly cited. The work cannot be changed in any way or used commercially. 
recruited 716 patients (66\% generalized, $27 \%$ unclassified) who were randomized to lamotrigine, topiramate, or valproate. Data from the SANAD trial therefore provide a unique opportunity to investigate outcomes after a first treatment failure and clinical factors that influence these outcomes.

METHODS Outcomes. Outcomes are time to 12-month remission and time to second treatment failure, both measured from date of first treatment failure due to inadequate seizure control or unacceptable adverse events. Twelve-month remission is defined as time to 365 days of continuous seizure freedom. Second treatment failure is defined as withdrawal of a treatment, or addition of a drug, which may be due to inadequate seizure control, unacceptable adverse events, or both.

Patients and procedures. The methods for the SANAD trial have been published elsewhere. ${ }^{1,2}$ In summary, patients were eligible for inclusion if, in the previous year, they had a history of at least 2 clinically definite unprovoked seizures and they were at least 5 years old. Patients were recruited into SANAD arm A if the recruiting clinician considered carbamazepine to be the optimal standard treatment option. Patients were then allocated to start treatment with carbamazepine, gabapentin, lamotrigine, oxcarbazepine, or topiramate. Patients were eligible for inclusion in arm $B$ if the recruiting clinician regarded valproate the standard treatment option. Participants were randomly allocated to valproate, lamotrigine, or topiramate. One protocol was used for both arms $\mathrm{A}$ and $\mathrm{B}$.

Prognostic modeling. Our approach was similar to that published previously, ${ }^{10}$ and our list of potential prognostic factors included the following: sex, history of at least one febrile seizure (associated with fever but without evidence of intracranial infection or defined cause), first-degree relative with epilepsy, age at first treatment failure, time on randomized (first) treatment, neurologic insult, total number of tonic-clonic seizures ever at first treatment failure, reason for first treatment failure (inadequate seizure control or adverse effects), seizure type, epilepsy type, EEG result at entry into the SANAD trial, and CT or MRI result at entry into SANAD. Randomized drug was also considered as a prognostic factor to determine whether the outcomes were influenced by first drug.

Patients were classified as having neurologic insult if they had learning disabilities (a history of requiring a remedial or additional teacher in more than 2 subjects, or who underwent a "statement of their education needs," or attended a special school) or neurologic deficit (localizing neurologic signs resulting in functional impairment). EEG was classified as normal, not done, nonspecific abnormality, or epileptiform abnormality (focal or generalized spikes or spike and slow-wave activity). Seizure types were classified according to the International League Against Epilepsy seizure classification. ${ }^{12}$ Epilepsy type was classified as focal, generalized, or unclassified with the unclassified category representing uncertainty between focal-onset and generalized-onset seizures.

Analyses, adjusted for multiple variables using Cox proportional hazards modeling, determined variables associated with a greater likelihood of achieving the outcomes. Variable centering was used to diminish multicollinearity: binary independent variables were coded $+1 / 2$ and $-1 / 2$ rather than 1 and 0 ; categorical independent variables were "dummy-coded" as usual, but instead of coding each response as 1 and 0 , the values $1-1 / \mathrm{m}$ and $-1 / \mathrm{m}$ were used, where $\mathrm{m}$ is the number of categories. ${ }^{13}$ Parsimonious multivariable models were produced with variable selection via backward elimination with Akaike Information Criterion. ${ }^{14}$ The full model included all factors listed above.

All continuous variables were investigated using log and fractional polynomial transformations; selection was made via the Akaike Information Criterion. ${ }^{15-18}$ Continuous variable results are presented as post hoc defined categorical variables with categories chosen according to knot positions for a spline model fit to the data. ${ }^{19}$ Schoenfeld ${ }^{20}$ residual plots and incorporation of timedependent covariate effects were used to investigate the proportional hazards assumption of the Cox model.

Kaplan-Meier curves were produced to show the unadjusted time to 12-month remission and time to second treatment failure after a first treatment failure. The discriminatory power and predictive accuracy of the models were assessed, as a method of internal validation, by the $\mathrm{C}$ statistic. This measures the proportion of patient pairs in which the predictions and outcomes are concordant. ${ }^{21}$ To assess the effect of factors on the different reasons for second treatment failure, subgroup analyses were performed: factors that predict the failure outcome were considered for patients whose first treatment failed due to inadequate seizure control and for patients whose first treatment failed due to unacceptable adverse effects.

Standard protocol approvals, registrations, and patient consents. The SANAD trial received appropriate multicenter and local ethics and research committee approvals, and was managed according to the Medical Research Council's Good Clinical Practice Guidelines. Patients gave informed written consent to inclusion and to long-term follow-up. SANAD is registered as an International Standard Randomized Controlled Trial, number ISRCTN38354748.

RESULTS Figure 1 shows the flow of the 2,627 patients recruited into arms $\mathrm{A}$ and $\mathrm{B}$ of the SANAD trial. After a first treatment failure, 147 patients did not start another AED. These patients were included in the analysis of time to 12 -month remission but excluded from the analysis of time to second treatment failure. Of these patients, 130 had withdrawn from their randomized treatment because of unacceptable adverse events, and 17 had withdrawn because of inadequate seizure control.

A total of 1,065 patients were included in the analysis of time to 12-month remission, of whom 535 had a 12-month remission. Nine hundred twenty-eight patients were included in the analysis of time to second treatment failure, of whom 356 had a second treatment failure. Characteristics of patients included in time to 12-month remission analysis are summarized in table 1. Data were similar for time to second treatment failure (not shown).

Table e-1 (on the Neurology ${ }^{\circledR}$ Web site at Neurology.org) summarizes the treatments that patients were switched to after the first failure. After treatment failure on carbamazepine, oxcarbazepine, or valproate, the most likely treatment switch was to lamotrigine. In patients for whom gabapentin, lamotrigine, and topiramate failed, the most likely switch was to carbamazepine. 


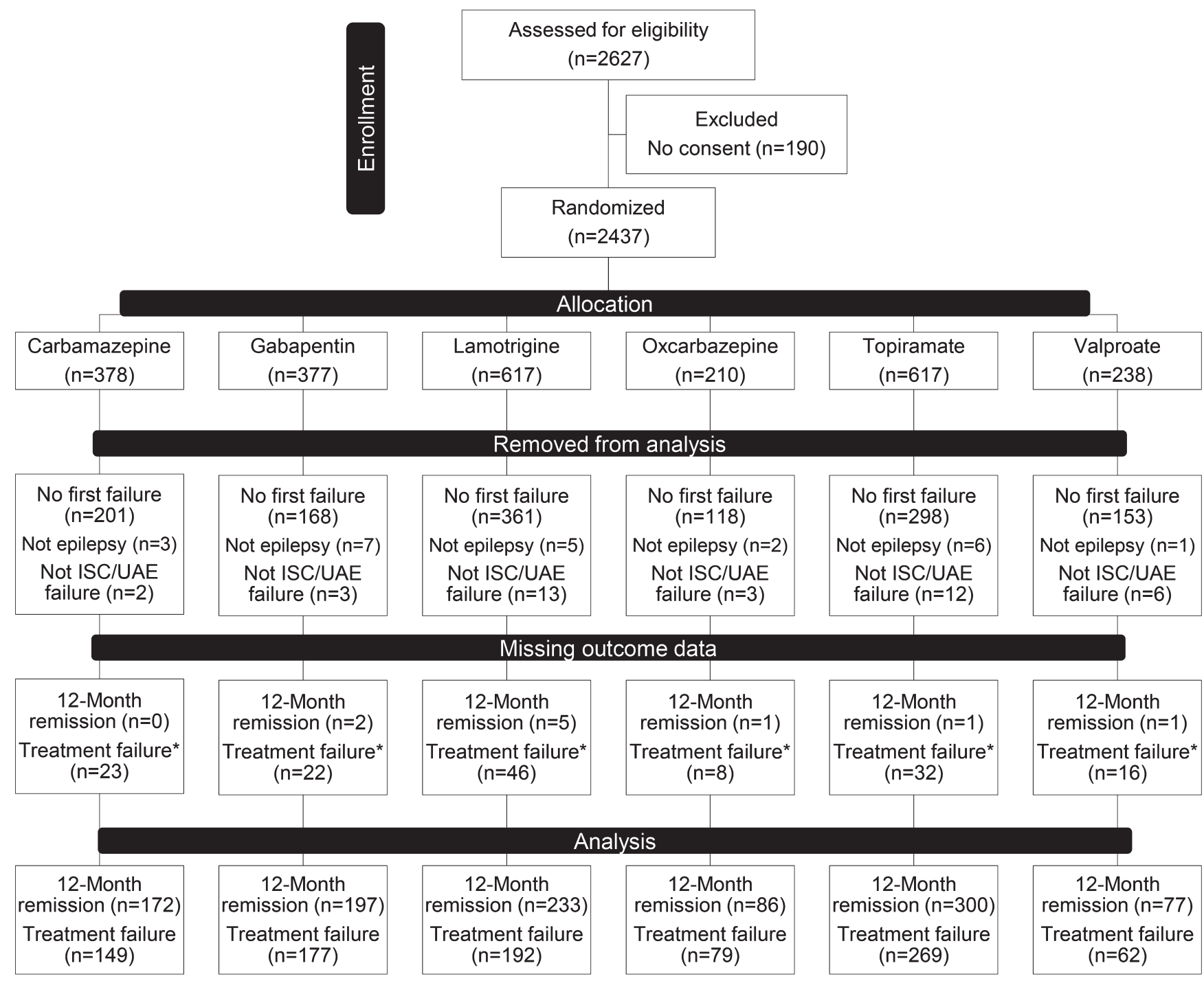

Carbamazepine, gabapentin, lamotrigine, oxcarbazepine, and topiramate were randomized drugs in arm A. Lamotrigine, topiramate, and valproate were randomized drugs in arm B. For this reason, the numbers randomized varied across the drugs. In addition, oxcarbazepine was only added to arm A midway through the trial, hence fewer patients were randomized to it. *No second drug information available. ISC = inadequate seizure control; $\mathrm{UAE}=$ unacceptable adverse event.

Time to 12-month remission after a first treatment failure. The Kaplan-Meier curve for time to 12month remission after a first treatment failure for any reason can be seen in figure e-1. Approximately $30 \%$ of patients failing their first treatment achieved 12-month remission immediately after the first failure. At 5 years, overall, $70 \%$ of patients achieved a 12-month remission: 65\% with a first failure due to inadequate seizure control and $80 \%$ with a first treatment failure due to unacceptable adverse events.

Results for the parsimonious multivariable model for time to 12-month remission after first treatment failure are summarized in table 2 (see table e- 2 for regression coefficients and standard errors.) The $\mathrm{C}$ statistic for the model was 0.6. This means that, for a random pair of patients, the probability of the patient who achieved 12-month remission first having the shorter predicted probability of achieving 12 -month remission is $60 \%$, which suggests low predictive utility. ${ }^{22,23}$

Initial AED was not significant univariately and was not included in the multivariable model. Patients whose first treatment failed due to unacceptable adverse events were more likely to achieve 12-month remission after a first treatment failure than those whose treatment failed due to inadequate seizure control. Twelve-month remission was more likely in men than in women, more likely in patients older than 45 years than those younger than 11 years, and more likely for patients with a shorter duration of treatment with the first AED. 
Table 1 Patient characteristics for patients with a first treatment failure

Males, n (\%)

Febrile seizure history, $\mathrm{n}(\%)$

First-degree relative, $\mathrm{n}(\%)$

Neurologic insult, ${ }^{a} \mathrm{n}(\%)$

Epilepsy type, $n(\%)$

\section{Focal}

Generalized

Unclassified

Seizure type, $\mathrm{n}(\%)$

Simple or complex partial with secondary generalized seizures

Simple or complex partial only

Generalized tonic-clonic seizures only

Absence seizures

Myoclonic or absence seizures with tonic-clonic seizures

Tonic-clonic seizures, uncertain if focal or generalized

Other

EEG, $n$ (\%)

\section{Normal}

Nonspecific abnormality

Epileptiform abnormality

Not done

CT/MRI, n (\%)

\section{Normal}

Abnormal

Not done

Age at first treatment failure, $y$, median (IQR)

Time on randomized treatment at treatment failure, mo, median (IQR)

Total no. of tonic-clonic seizures at first treatment failure, median (IQR)

Reason for first treatment failure, $n(\%)$

Inadequate seizure control

Unacceptable adverse events

AED at randomization

\begin{tabular}{|c|c|c|c|c|c|c|}
\hline $\begin{array}{l}\text { CBZ } \\
\text { (n = 172) }\end{array}$ & $\begin{array}{l}\text { GBP } \\
(n=197)\end{array}$ & $\begin{array}{l}\text { LTG } \\
(n=233)\end{array}$ & $\begin{array}{l}\text { OXC } \\
(n=86)\end{array}$ & $\begin{array}{l}\text { TPM } \\
(n=300)\end{array}$ & $\begin{array}{l}\text { VPS } \\
(\mathrm{n}=77)\end{array}$ & $\begin{array}{l}\text { Total } \\
(n=1,065)\end{array}$ \\
\hline $93(54)$ & 97 (49) & $133(57)$ & 41 (48) & $158(53)$ & 48 (62) & $570(54)$ \\
\hline $13(8)$ & $6(3)$ & $20(9)$ & $3(3)$ & $19(6)$ & $3(4)$ & $64(6)$ \\
\hline 17 (10) & 23 (12) & 37 (16) & $7(8)$ & 39 (13) & 12 (16) & 135 (13) \\
\hline 19 (11) & $22(11)$ & 33 (14) & 7 (8) & 41 (14) & 8 (10) & $130(12)$ \\
\hline $158(92)$ & $183(93)$ & $135(58)$ & 78 (91) & $174(58)$ & 10 (13) & $738(69)$ \\
\hline $2(1)$ & $2(1)$ & 66 (28) & $3(3)$ & 81 (27) & 47 (61) & 201 (19) \\
\hline $12(7)$ & $12(6)$ & 32 (14) & $5(6)$ & 45 (15) & 20 (26) & 126 (12) \\
\hline 94 (55) & 117 (59) & 87 (37) & $51(60)$ & 94 (31) & 7 (9) & $450(43)$ \\
\hline 64 (37) & 66 (34) & 48 (21) & 27 (31) & 78 (26) & $3(4)$ & 286 (27) \\
\hline $2(1)$ & 1 (1) & $16(7)$ & $3(3)$ & 26 (9) & 20 (26) & $68(6)$ \\
\hline $0(0)$ & $0(0)$ & $20(8)$ & $0(0)$ & $23(8)$ & 12 (16) & $55(5)$ \\
\hline $0(0)$ & $0(0)$ & 25 (11) & $0(0)$ & $28(9)$ & 14 (18) & $67(6)$ \\
\hline $10(6)$ & $10(5)$ & 29 (13) & $4(5)$ & 38 (13) & 17 (22) & 108 (10) \\
\hline $2(1)$ & $3(1)$ & 8 (3) & 1 (1) & $13(4)$ & $4(5)$ & 31 (3) \\
\hline 79 (46) & $96(49)$ & 73 (31) & $34(40)$ & 87 (29) & 24 (31) & 393 (37) \\
\hline 29 (17) & 29 (15) & 30 (13) & 17 (20) & 39 (13) & $8(10)$ & $152(14)$ \\
\hline 51 (30) & 62 (31) & 110 (47) & 24 (27) & 144 (48) & 42 (55) & $433(41)$ \\
\hline $13(7)$ & $10(5)$ & $20(9)$ & 11 (13) & 30 (10) & $3(4)$ & 87 (8) \\
\hline 94 (55) & $130(66)$ & $117(50)$ & 53 (62) & $146(49)$ & 40 (52) & $580(54)$ \\
\hline 50 (29) & 43 (22) & 44 (19) & 22 (25) & 69 (23) & $5(6)$ & 233 (22) \\
\hline $28(16)$ & 24 (12) & 72 (31) & 11 (13) & 85 (28) & 32 (42) & 252 (24) \\
\hline $39(27,53)$ & $36(25,49)$ & $26(16,43)$ & $38(26,57)$ & $30(17,45)$ & $20(16,29)$ & $32(20,46)$ \\
\hline $\begin{array}{l}5.3(1.2, \\
13.9)\end{array}$ & $\begin{array}{l}6.5(2.8 \\
12.0)\end{array}$ & $\begin{array}{l}6.7(1.6 \\
16.6)\end{array}$ & $\begin{array}{l}3.5(1.1 \\
10.8)\end{array}$ & $\begin{array}{l}4.9 \text { (2.0, } \\
12.0)\end{array}$ & $\begin{array}{l}5.1(1.7 \\
11.7)\end{array}$ & $5.5(1.7,12.9)$ \\
\hline $2(0,5)$ & $2(0,5)$ & $2(0,6)$ & $2(0,5)$ & $2(0,5)$ & $3(1,6)$ & $2(0,5)$ \\
\hline 61 (35) & $126(64)$ & $127(55)$ & 35 (41) & $126(42)$ & 32 (42) & 507 (48) \\
\hline 111 (65) & 71 (36) & $106(45)$ & $51(59)$ & 174 (58) & 45 (58) & $558(52)$ \\
\hline
\end{tabular}

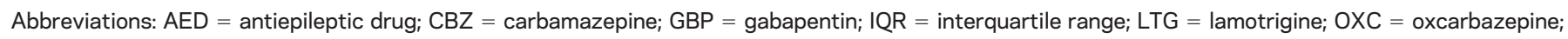
TPM = topiramate; VPS = valproate.

a For example, includes hemiparesis and learning difficulty.

The occurrence of tonic-clonic seizures (primary or secondary generalized) on first AED was associated with a lower probability of subsequent 12-month remission. The combination of seizure types that patients had experienced on entry to the SANAD trial was also of prognostic importance: 12-month remission was more likely in patients with generalized tonic-clonic seizures than in those with focal and secondary generalized seizures, while the latter were more likely to have a 12-month remission than those with focal seizures without secondary generalization.

To illustrate the range of remission rates predicted by the multivariable model, figure 2 shows estimates of the proportion of patients achieving 12-month remission 1 and 3 years after first treatment failure. Patients were assumed to be male, to have no 
Table 2 Multivariable results for time to 12-month remission after first treatment failure

Comparison
Sex
Female
Male
Age, y
$\leq 10$
$11-20$
$21-30$
$31-45$
$46-70$
$>70$

Time on randomized treatment at first treatment failure, mo

1

2

3,6

7,12

13,36

$>36$

Neurologic insult

Absent

Present

Total no. of tonic-clonic seizures before first treatment failure

0

1

2

3-4

5-6

7-10

11-20

$>20$

Reason for treatment failure

Inadequate seizure control

Unacceptable adverse events

Seizure type

Simple or complex partial with secondary generalized seizures

Simple or complex partial only

Generalized tonic-clonic seizures only

Absence seizures

Myoclonic or absence seizures with tonic-clonic seizures

Tonic-clonic seizures, uncertain if focal or generalized

Other

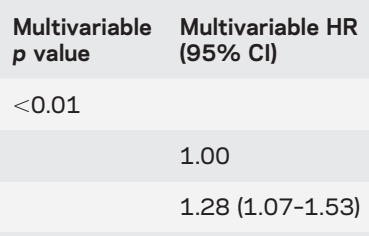

$<0.01$

1.00

$0.98(0.95-1.00)$

0.96 (0.91-1.02)

$1.00(0.90-1.10)$

$1.28(1.05-1.58)$

$2.25(1.43-3.53)$

$<0.01$

1.00

$0.80(0.70-0.93)$

$0.77(0.65-0.92)$

$0.76(0.63-0.91)$

$0.75(0.62-0.91)$

$0.75(0.62-0.91)$

0.14

1.00

$0.80(0.59-1.07)$

$<0.01$

1.00

$0.96(0.93-0.98)$

$0.93(0.89-0.98)$

0.91 (0.85-0.97)

$0.89(0.82-0.96)$

$0.87(0.79-0.96)$

0.84 (0.74-0.95)

$0.66(0.50-0.88)$

$<0.01$

1.00

$1.70(1.40-2.06)$

$-$

1.00

0.01

$0.70(0.53-0.93)$

$0.03 \quad 1.67(1.04-2.70)$

$0.71 \quad 1.11(0.64-1.92)$

$0.87 \quad 0.96(0.59-1.58)$

$0.70 \quad 1.08(0.75-1.55)$

$0.87(0.49-1.53)$ neurologic insult, and to have spent 6 months on the randomized treatment at treatment failure. The other variables were altered according to categories of interest (e.g., age as 10 and 40 years). Numeric results can be seen in table e-3.

Time to second treatment failure after a first treatment failure. The Kaplan-Meier curve for time to second treatment failure after a first for any reason can be seen in figure e-2. Of patients whose first treatment failed, approximately $20 \%$ had another treatment failure by 6 months and approximately $25 \%$ by 1 year. At 3 years, overall, approximately $40 \%$ had a second treatment failure, while $45 \%$ with a first failure due to inadequate seizure control and 30\% with a treatment failure due to unacceptable adverse events had a second treatment failure by 3 years after a first treatment failure.

Table 3 shows results for the 3 separate multivariable models. (See table e- 4 for regression coefficients and standard errors.) The first is for time to second treatment failure irrespective of the reason for first treatment failure (inadequate seizure control or adverse events). This model includes number of tonic-clonic seizures before first failure, CT/MRI results, and reason for first failure. The $\mathrm{C}$ statistic for this model is 0.6. This means that, for a random pair of patients, the probability of the patient who had a second treatment first having the shorter predicted probability of second treatment failure is $60 \%$, which suggests low predictive utility. Once again, type of initial AED was not significantly associated with the outcome. Risk estimates for combinations of risk factors can be seen in table e-5.

The second model is for patients with a first failure due to inadequate seizure control and the third for patients with a first failure due to adverse events. As might be anticipated, these models are quite different. The model including patients with a first failure due to inadequate seizure control includes age and CT/MRI results, and has a $\mathrm{C}$ statistic of 0.6, suggesting low predictive utility. The model including patients with a first failure due to adverse effects includes number of tonic-clonic seizures before first treatment failure and EEG, and has a $\mathrm{C}$ statistic of 0.6 , suggesting low predictive utility. Risk estimates for stratified groups of patients whose first treatment failed due to unacceptable adverse events can be seen in table e-6.

DISCUSSION Numerous factors contribute to the multivariable model for 12-month remission, highlighting the heterogeneity of epilepsy, but also highlighting the prognostic importance of information collected in routine clinical practice. The model is able to stratify patients, and the estimates for 12-month 


\begin{tabular}{|lll|}
\hline Table $2 \quad$ Continued & & \\
Comparison & $\begin{array}{l}\text { Multivariable } \\
\mathbf{p} \text { value }\end{array}$ & $\begin{array}{l}\text { Multivariable HR } \\
(95 \% \mathrm{Cl})\end{array}$ \\
CT/MRI & & \\
Normal & - & 1.00 \\
Abnormal & 0.11 & $0.83(0.66-1.06)$ \\
Not done & 0.12 & $1.20(0.96-1.50)$ \\
\hline
\end{tabular}

Abbreviations: $\mathrm{Cl}=$ confidence interval; $\mathrm{HR}=$ hazard ratio.

remission rates could be used in routine clinical practice to inform likely outcomes.

The model published here has a number of similarities to the model for time to 12-month remission from randomization in SANAD. ${ }^{10,11}$ Unsurprisingly, reason for first treatment failure is of prognostic importance as is duration of first treatment. Early treatment failure is more likely to be due to adverse effects, so duration of first treatment and reason for first treatment failure are likely to be correlated, but nonetheless, both factors were included in the multivariable model.

While broad epilepsy type (focal vs generalized) was not included in the multivariable model, this was, in part, accounted for by seizure type, which was included. Also, the occurrence of tonic-clonic seizures (focal or generalized in onset) on first treatment was prognostic as was age.

The EEG result was not associated with 12-month remission, which is surprising because EEG abnormality was found to be associated with seizure recurrence risk in other studies. ${ }^{24,25}$ The most likely explanation is that the EEG data used were collected at entry into SANAD. Future studies assessing outcome after a first treatment failure need to undertake EEGs at that time point to assess their prognostic importance.

We also found that 12-month remission was more likely in men than in women, which was also established when the SANAD arm A data were modeled for time to 12-month remission from initiating treatment (randomization). ${ }^{10}$ This finding remains mostly unexplained because there was no significant difference in the way men and women were dosed, and it is unclear whether this finding represents differences in the way that men and women are managed, or whether this represents an underlying important biological difference between the sexes that influences drug response. A study in children also found sex to be significant; the authors showed that discontinuation of AEDs in seizure-free children was less likely to succeed if the patient was female, had an abnormal neurologic examination, seizure onset of less than 120 months, and had focal seizures. ${ }^{26}$

It is important to note that first AED was not included in the model, highlighting that clinical factors have a more important impact on outcome than choice of first drug. This should not be surprising because differences found among drugs have been small and drug development programs have retreated to finding noninferiority as opposed to superiority for new treatments. ${ }^{27,28}$

For time to second treatment failure after a first treatment failure, we have produced 3 models. The first examines time to a second treatment failure irrespective of the reason for a first treatment failure. The models assessing subgroups according to reason for first treatment failure give quite different results.

The SANAD trial was not designed to address the question considered here. Although patients were followed up regardless of outcome, it is possible that data collection for the outcomes examined in this report may not have been as complete or accurate as for the primary outcomes in the trial. Some have criticized the fact that SANAD recruited a heterogeneous group of patients rather than specific seizure types or epilepsy syndromes ${ }^{29,30}$; however, the strength of this approach is illustrated in this report and in our previous reports modeling data from SANAD. ${ }^{10,11}$

SANAD was an unblinded trial, which could have influenced outcome assessment; for example, decisions as to whether a treatment had failed, although examination of dosing data indicates that reasonable doses were tried before a decision was made that treatment had failed. While randomized controlled trials are the best methodology for assessing treatment effects, they might recruit a selected population, which might bias estimates of prognosis.

Participants were seen predominantly by neurologists experienced at identifying and classifying seizures, but a further challenge in outpatient-based studies of seizures and epilepsy is that seizures are reported to the clinician by the patient and it is possible that patients underreport the occurrence of seizures. Validating patient reporting in an outpatient population with infrequent seizures is difficult and to date has not been done.

The predictive ability of the models presented within this report is low, as illustrated by the low C statistics for each model. While there is increasing interest in stratified medicine and pharmacogenetics, it remains unknown as to whether genetic factors might account for some of the unexplained variability in our predictive models. In the future, they may help to improve the predictive accuracy of the models.

The models presented here are the first to consider chance of remission and risk of second treatment failure after a first treatment failure. While we have 


\begin{tabular}{ccccc} 
Age & No. T-C & Reason & S. Type & CT/MRI \\
10 & 0 & ISC & SCGTC & Norm \\
10 & 0 & ISC & SCGTC & Ab \\
10 & 0 & ISC & GenT-C & Norm \\
10 & 0 & ISC & Gen T-C & Ab \\
10 & 0 & UAE & SCGTC & Norm \\
10 & 0 & UAE & SCGTC & Ab \\
10 & 0 & UAE & GenT-C & Norm \\
10 & 0 & UAE & GenT-C & Ab \\
\hline 10 & 10 & ISC & SCGTC & Norm \\
10 & 10 & ISC & SCGTC & Ab \\
10 & 10 & ISC & GenT-C & Norm \\
10 & 10 & ISC & GenT-C & Ab \\
10 & 10 & UAE & SCGTC & Norm \\
10 & 10 & UAE & SCGTC & Ab \\
10 & 10 & UAE & Gen T-C & Norm \\
10 & 10 & UAE & GenT-C & Ab \\
\hline 40 & 0 & ISC & SCGTC & Norm \\
40 & 0 & ISC & SCGTC & Ab \\
40 & 0 & ISC & GenT-C & Norm \\
40 & 0 & ISC & GenT-C & Ab \\
40 & 0 & UAE & SCGTC & Norm \\
40 & 0 & UAE & SCGTC & Ab \\
40 & 0 & UAE & Gen T-C & Norm \\
40 & 0 & UAE & GenT-C & Ab \\
\hline 40 & 10 & ISC & SCGTC & Norm \\
40 & 10 & ISC & SCGTC & Ab \\
40 & 10 & ISC & Gen T-C & Norm \\
40 & 10 & ISC & Gen T-C & Ab \\
40 & 10 & UAE & SCGTC & Norm \\
40 & 10 & UAE & SCGTC & Ab \\
$4 A E$ & Gen T-C & Ab
\end{tabular}
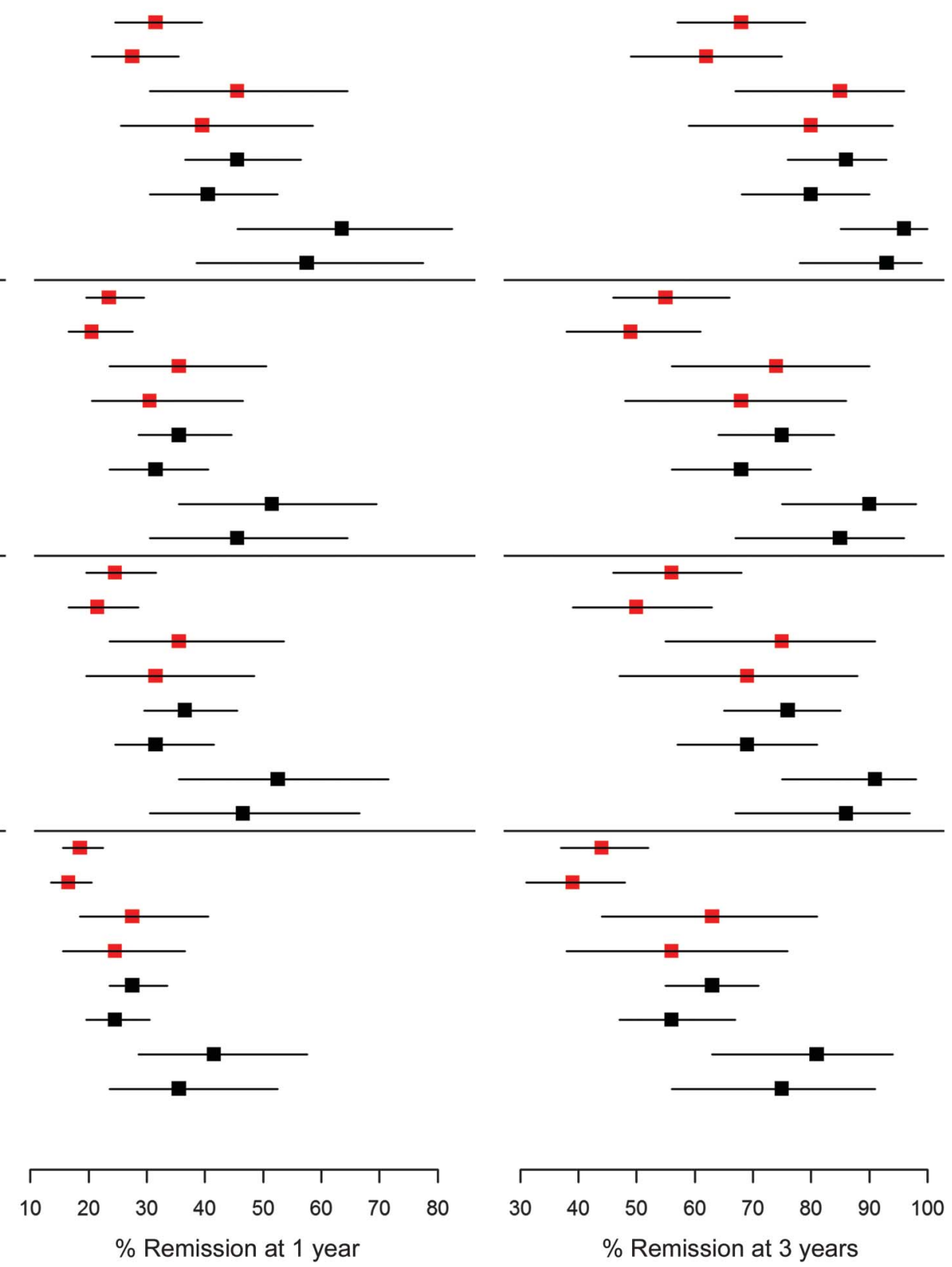

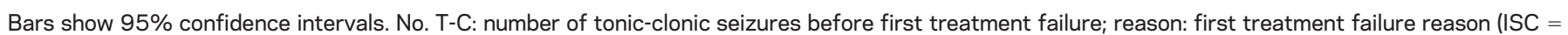

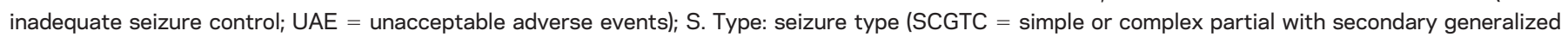

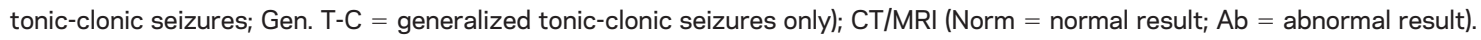

presented a number of models that may inform patient counseling and treatment decisions, ideally these models require validation in other similar datasets, although few such datasets exist. In addition, the mechanisms by which the clinical factors identified in this report influence outcome remain poorly understood and require further assessment.

This article presents the first report of prognostic modeling for time to 12 -month remission and time to second treatment failure, both after a first treatment failure. Our results highlight the heterogeneity of outcome in epilepsy and the complex interplay among the factors that influence it. Patients with differing risks of 12 months of remission and treatment failure could be identified at the point in time when the initial AED treatment failed. Results will improve outcome prediction for patients, and allow better stratification of patients, including the identification of patients more likely to have poor treatment outcome for whom more intensive follow-up may be required. Similarly, the models may also aid in the identification of patients with poorer 
Table 3 Multivariable results for time to second treatment failure after first treatment failure

\begin{tabular}{|c|c|c|c|c|}
\hline \multirow[b]{2}{*}{ Comparison } & \multirow[b]{2}{*}{ Multivariable $p$ value (overall) } & \multicolumn{3}{|c|}{$\begin{array}{l}\text { Multivariable HR }(95 \% \mathrm{Cl}) \text { by first treatment failure } \\
\text { reason }\end{array}$} \\
\hline & & Overall $(n=928)$ & ISC $(n=499)$ & UAE (n = 429) \\
\hline$\leq 10$ & & & 1.00 & \\
\hline $11-20$ & & & $0.95(0.88-1.01)$ & \\
\hline $31-45$ & & & $0.88(0.75-1.03)$ & \\
\hline $46-70$ & & & $0.85(0.69-1.04)$ & \\
\hline$>70$ & & & $0.83(0.65-1.05)$ & \\
\hline Total no. of tonic-clonic seizures before first treatment failure & 0.06 & & NA & NA \\
\hline $5-20$ & & $1.01(1.00-1.02)$ & & \\
\hline$>20$ & & $1.77(0.97-3.21)$ & & \\
\hline Time on randomized treatment at first treatment failure, mo & NA & NA & NA & \\
\hline 1 & & & & 1.00 \\
\hline 2 & & & & $1.01(1.00-1.02)$ \\
\hline $3-6$ & & & & $1.04(1.01-1.06)$ \\
\hline 7-12 & & & & $1.09(1.03-1.16)$ \\
\hline $13-36$ & & & & $1.27(1.08-1.49)$ \\
\hline$>36$ & & & & $2.71(1.39-5.31)$ \\
\hline Epileptiform abnormality & & & & $0.66(0.45-0.97)$ \\
\hline Nonspecific abnormality & & & & $1.09(0.69-1.74)$ \\
\hline No results & & & & $1.23(0.68-2.24)$ \\
\hline CT/MRI & & & & NA \\
\hline Normal & - & 1.00 & 1.00 & \\
\hline Abnormal & 0.01 & $0.97(0.75-1.26)$ & $0.99(0.71-1.39)$ & \\
\hline Not done & 0.82 & $0.65(0.49-0.88)$ & $0.48(0.32-0.71)$ & \\
\hline C statistic & & 0.6 & 0.6 & 0.6 \\
\hline
\end{tabular}

Abbreviations: $\mathrm{Cl}=$ confidence interval; $\mathrm{HR}=$ hazard ratio; ISC = inadequate seizure control; $\mathrm{NA}=$ not applicable; UAE = unacceptable adverse event.

seizure control outcomes who might be eligible to participate in future trials of new treatments, for example surgical treatments such as deep brain stimulation, which might carry greater risk than drug treatment.

\section{AUTHOR CONTRIBUTIONS}

L.J.B. performed all the statistical analyses, drafted and revised the manuscript. C.T.S. drafted and revised the manuscript. S.D. calculated the treatment failure outcome, drafted and revised the manuscript. A.G.M. drafted and revised the manuscript and was principal investigator of the SANAD study.

\section{STUDY FUNDING}

This report presents independent research funded by the National Institute for Health Research (NIHR) under its Programme Grants for Applied Research Programme (grant RP-PG-0606-1062). The views expressed are those of the author(s) and not necessarily those of the NHS, the NIHR, or the Department of Health. 


\section{DISCLOSURE}

The authors report no disclosures relevant to the manuscript. Go to Neurology.org for full disclosures.

Received September 10, 2013. Accepted in final form April 28, 2014

\section{REFERENCES}

1. Marson AG, Al-Kharusi AM, Alwaidh M, et al. The SANAD study of effectiveness of carbamazepine, gabapentin, lamotrigine, oxcarbazepine, or topiramate for treatment of partial epilepsy: an unblinded randomised controlled trial. Lancet 2007;369:1000-1015.

2. Marson AG, Al-Kharusi AM, Alwaidh M, et al. The SAN$\mathrm{AD}$ study of effectiveness of valproate, lamotrigine, or topiramate for generalised and unclassifiable epilepsy: an unblinded randomised controlled trial. Lancet 2007;369: 1016-1026.

3. Tudur Smith C, Marson AG, Chadwick DW, Williamson PR. Multiple treatment comparisons in epilepsy monotherapy trials. Trials 2007;8:34.

4. Kwan P, Arzimanoglou A, Berg AT, et al. Definition of drug resistant epilepsy: consensus proposal by the ad hoc Task Force of the ILAE Commission on Therapeutic Strategies. Epilepsia 2010;51:1069-1077.

5. Gilioli I, Vignoli A, Visani E, et al. Focal epilepsies in adult patients attending two epilepsy centers: classification of drug-resistance, assessment of risk factors, and usefulness of "new” antiepileptic drugs. Epilepsia 2012; 53:733-740.

6. Cockerell OC, Johnson AL, Sander JW, Hart YM, Shorvon SD. Remission of epilepsy: results from the National General Practice Study of Epilepsy. Lancet 1995;346:140-144.

7. Randomised study of antiepileptic drug withdrawal in patients in remission. Medical Research Council Antiepileptic Drug Withdrawal Study Group. Lancet 1991;337: 1175-1180.

8. Chadwick D, Taylor J, Johnson T. Outcomes after seizure recurrence in people with well-controlled epilepsy and the factors that influence it. The MRC Antiepileptic Drug Withdrawal Group. Epilepsia 1996;37:1043-1050.

9. Marson A, Jacoby A, Johnson A, Kim L, Gamble C, Chadwick D. Immediate versus deferred antiepileptic drug treatment for early epilepsy and single seizures: a randomised controlled trial. Lancet 2005;365:2007-2013.

10. Bonnett LJ, Tudur Smith C, Smith D, Williamson P, Chadwick D, Marson AG. Prognostic factors for time to treatment failure and time to 12 months of remission for patients with focal epilepsy: post-hoc, subgroup analyses of data from the SANAD trial. Lancet Neurol 2012;11: 331-340.

11. Bonnett LJ, Tudur Smith C, Smith D, Williamson PR, Chadwick D, Marson AG. Time to 12-month remission and treatment failure for generalised and unclassified epilepsy. J Neurol Neurosurg Psychiatry 2014;85: 603-610.

12. Proposal for revised clinical and electroencephalographic classification of epileptic seizures. From the Commission on Classification and Terminology of the Internationa League Against Epilepsy. Epilepsia 1981;22:489-501.
13. Kraemer HC, Blasey CM. Centring in regression analyses: a strategy to prevent errors in statistical inference. Int J Methods Psychiatr Res 2004;13:141-151.

14. Akaike H. Information theory as an extension of the maximum likelihood principle. In: Petrov BN, Csaki F, editors. Second International Symposium on Information Theory. Budapest: Akadémiai Kiadó; 1973:267-281.

15. Royston P, Ambler G, Sauerbrei W. The use of fractional polynomials to model continuous risk variables in epidemiology. Int J Epidemiol 1999;28:964-974.

16. Royston P, Altman DG. Regression using fractional polynomials of continuous covariates: parsimonious parametric modeling. Appl Stat 1994;43:429-467.

17. Royston P, Sauerbrei W. Multivariable Model-Building: A Pragmatic Approach to Regression Analysis Based on Fractional Polynomials for Modelling Continuous Variables. Hoboken, NJ: Wiley; 2008.

18. Royston P, Sauerbrei W. Building multivariable regression models with continuous covariates in clinical epidemiologywith an emphasis on fractional polynomials. Methods Inf Med 2005;44:561-571.

19. Stone CJ. Comment: generalized additive models. Stat Sci 1986;1:3.

20. Schoenfeld D. Partial residuals for the proportional hazards regression-model. Biometrika 1982;69:239-241.

21. Harrell FE, Lee KL, Mark DB. Multivariable prognostic models: issues in developing models, evaluating assumptions and adequacy, and measuring and reducing errors. Stat Med 1996;15:361-387.

22. Hosmer DW, Lemeshow S. Applied Logistic Regression, 2nd ed. New York: John Wiley \& Sons; 2000.

23. Harrell FE Jr. Regression Modeling Stategies: With Applications to Linear Models, Logistic Regression, and Survival Analysis. New York: Springer; 2001.

24. Kim LG, Johnson TL, Marson AG, Chadwick DW; MRC MESS Study Group. Prediction of risk of seizure recurrence after a single seizure and early epilepsy: further results from the MESS trial. Lancet Neurol 2006;5:317-322.

25. Berg AT, Shinnar S. The risk of seizure recurrence following a first unprovoked seizure: a quantitative review. Neurology 1991;41:965-972.

26. Dooley J, Gordon K, Camfield P, Camfield C, Smith E. Discontinuation of anticonvulsant therapy in children free of seizures for 1 year: a prospective study. Neurology 1996;46:969-974.

27. Brodie MJ, Perucca E, Ryvlin P, Ben-Menachem E, Meencke HJ; Levetiracetam Monotherapy Study Group. Comparison of levetiracetam and controlled-release carbamazepine in newly diagnosed epilepsy. Neurology 2007;68:402-408.

28. Baulac M, Brodie M, Segieth J, Giorgi L. Comparison of zonisamide and carbamazepine monotherapy in adults with newly diagnosed partial epilepsy: results of a phase III, randomized, double-blind, non-inferiority trial. Lancet Neurol 2012;78:IN5-1.005.

29. Panayiotopoulos CP. Old versus new antiepileptic drugs: the SANAD study. Lancet 2007;370:313-314.

30. Panayiotopoulos CP. Evidence-based epileptology, randomized controlled trials, and SANAD: a critical clinical view. Epilepsia 2007;48:1268-1274. 


\section{Neurology}

\section{Treatment outcome after failure of a first antiepileptic drug}

Laura J. Bonnett, Catrin Tudur Smith, Sarah Donegan, et al. Neurology 2014;83;552-560 Published Online before print July 3, 2014

DOI 10.1212/WNL.0000000000000673

\section{This information is current as of July 3, 2014}

\section{Updated Information \& Services}

\section{Supplementary Material}

\section{References}

Subspecialty Collections

\section{Permissions \& Licensing}

\section{Reprints}

including high resolution figures, can be found at: http://n.neurology.org/content/83/6/552.full

Supplementary material can be found at: http://n.neurology.org/content/suppl/2014/07/03/WNL.0000000000000 673.DC1

This article cites 26 articles, 4 of which you can access for free at: http://n.neurology.org/content/83/6/552.full\#ref-list-1

This article, along with others on similar topics, appears in the following collection(s):

All Clinical trials

http://n.neurology.org/cgi/collection/all_clinical_trials

All Epilepsy/Seizures

http://n.neurology.org/cgi/collection/all_epilepsy_seizures

Natural history studies (prognosis)

http://n.neurology.org/cgi/collection/natural_history_studies_prognosis Prognosis

http://n.neurology.org/cgi/collection/prognosis

Information about reproducing this article in parts (figures,tables) or in its entirety can be found online at:

http://www.neurology.org/about/about_the_journal\#permissions

Information about ordering reprints can be found online: http://n.neurology.org/subscribers/advertise

Neurology ${ }^{\circledR}$ is the official journal of the American Academy of Neurology. Published continuously since 1951, it is now a weekly with 48 issues per year. Copyright @ 2014 American Academy of Neurology. All rights reserved. Print ISSN: 0028-3878. Online ISSN: 1526-632X.

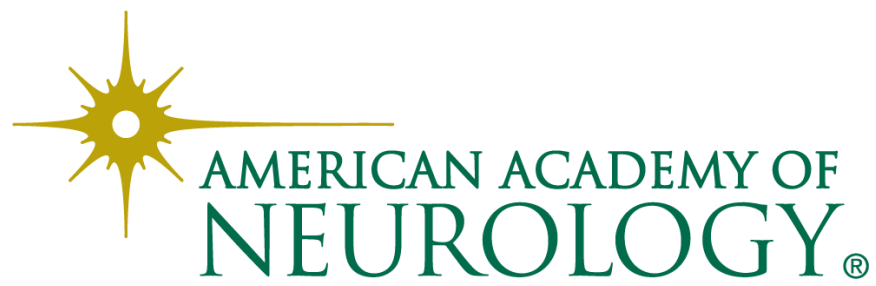

